\title{
Safety and Efficacy of Methylcobalamin in the Treatment of Peripheral Nerve Injuries and Diabetic Neuropathies - A Systematic Review
}

\section{Suresh Vyloppilli', Annamalai Thangavelu ${ }^{2 *}$, Sankar Vinod Vichattu ${ }^{3}$ and Shermil Sayd ${ }^{4}$}

${ }^{1}$ Ph.D. Scholar, Department of Oral and Maxillofacial Surgery, Rajah Muthiah

Dental College, Annamalai University, Chidambaram, Tamil Nadu, India

${ }^{2}$ Professor, Rajah Muthiah Dental College and Hospital, Department of Oral and

Maxillofacial Surgery, Tamil Nadu, India

${ }^{3}$ Professor and Head of the Department, Department of Oral and Maxillofacial

Surgery, Mar-Baselios Dental College, India

${ }^{4}$ Assistant Professor, Department of Oral and Maxillofacial Surgery, Kannur Dental

College, Anjarakandy, Kannur, India

*Corresponding Author: Annamalai Thangavelu, Professor, Department of Oral and Maxillofacial Surgery, Rajah Muthiah Dental College, Annamalai University, Chidambaram, Tamil Nadu, India.
Received: June 28, 2021

Published: July 21, 2021

(C) All rights are reserved by Annamalai

Thangavelu., et al.

\begin{abstract}
Background: Recovery of nerve injuries sprouts a complex recovery mechanism in the human body. Although unclear, the pathogenesis for peripheral neuropathy is multifactorial and is attributed to factors like herpes zoster or HIV infection, malignancies, diabetes, etc. Recently, the effect of high doses of methylcobalamin has been proved in both in-vitro and in-vivo experiments. We aim to review high doses of Methylcobalamin's impact on the neural regeneration.

Method: We conducted a systematic review of English literature in PubMed and related papers published over the internet.

Results and Conclusion: From the data obtained from the literature, it can be concluded that methylcobalamin plays an essential role in the human body, whose deficiency, combined with other factors, will cause neurological symptoms and decreased peripheral nerve repair. When given in mild doses for long term, no adverse effects have yet been reported, and those which were reported rarely were managed in the treatment period itself. Along with providing good results in the long term, it sits significantly better than other conventional methods for the management of neuropathies and associated neuropathic pain.
\end{abstract}

Keywords: Methycobalamine; Diabetic Neuropathy; Peripheral Nerve Injuries; Cyanocobalamine

\section{Introduction}

Following peripheral nerve injury, a complex reparative process eliminates the damage and restores the structure and function. Unlike cellular repair in other organs in the human body, peripheral nerve injury does not involve mitosis and cellular proliferation [1].
With advances in molecular biology, increasing attention is given to the process of peripheral nerve regeneration. The peripheral nerves can regenerate after injury on their own due to the activation of their intrinsic growth capacity [2]. The sciatic nerve is commonly used for studying peripheral nerve regeneration since it consists of both sensory and motor neurons $[3,4]$. 
According to the Center for Disease Control and Prevention, National Ambulatory Medical Care survey factsheet published in 2010 [5], peripheral neuropathy is the leading reason for neurologic consultations worldwide. Patients report symptoms ranging anywhere from tingling to loss of sensation [6]. Although unclear, the pathogenesis for peripheral neuropathy is multifactorial and is attributed to factors like herpes zoster or HIV infection, malignancies, diabetes, etc.

Studies have established that methylcobalamin (MeCbl) has a unique affinity for neural tissues [7]. All of the vitamin B12 is constituted by cobalt. The significant difference between them is incorporating various side chains like cyanide (-CN) for cyanocobalamin and methyl side chain methylcobalamin $\left(\mathrm{CH}_{3}\right)$. Substitution of the cyanide side chain with methyl sidechain increases the neural uptake of these compounds. They promote myelination and transport of the axonal cytoskeleton, thereby helping to maintain and regenerate peripheral nerves $[8,9]$. They are also characterized by more effective systemic or local delivery. Although the effect of methylcobalamin has been extensively reported in the literature, this regimen of treatment is not yet official in most of the countries, including the united states of America's food and drug administration [10]. It is most prevalent among Asian countries. But the topic of methylcobalamin and its effectiveness in the management of peripheral neuropathy is still controversial [11-13].

Recently, the effect of high doses of methylcobalamin has been proved in both in-vitro and in-vivo experiments [14]. In addition, a study by Okada., et al. demonstrated that high doses MeCbl can promote neuronal survival and neurite outgrowth in rat sciatic nerve injuries [15]. But, no human trials have yet been conducted on administering high dose MeCbl to humans [8].

\section{Materials and Methods}

A systematic review of the English literature was done in Pubmed and for related papers on the internet. All the available literature was used for the compilation of this review article. Bibliographies of already published articles were also examined for any related documents. None of the literature where the complete publication was not available was included in the study. Conference presentations, abstracts, and letters to the editors were also excluded. Further, the selected articles were scrutinized based on the data, whether belonging to modern medicine or not, and the status of their reviews, adherence to protocols and the selection was reduced to a handful that represented the most relevant data pertaining to this article.

\section{Mechanism of action of vitamin B12 induced neuropathy}

A lack of methylcobalamin due to the deficiency of vitamin B12 has been associated with significant neurological pathology [16] and has been attributed to hypomethylation in the central nervous system. Inhibition of the B12 -dependent enzyme methionine synthase results in a fall in the ratio of $\mathrm{S}$-adenosylmethionine (SAM) to $\mathrm{S}$-adenosylhomocysteine; the resultant deficiency in SAM affects the methionine conserving enzymes of the methionine cycles, therefore impairs the methylation reactions in the myelin sheath [17]. In patients with diabetic neuropathy, diabetes itself may be the reason for its occurrence or can be due to some anti-diabetic agents such as metformin [18]. The same can occur in patients with renal failure. A decreased serum thiocyanate clearance and increased cyanocobalamin synthesis indicate an elevation in the cyanide pool and is related to uraemic neuropathy. Methylcobalamin is considered to be used in the cyanide detoxication process via cyanocobalamin synthesis. Based on an in-vitro study, it is suggested that the altered membrane properties through SAM-mediated methylation are the primary mechanism of the neuroprotective effect of methylcobalamin in multiple types of CNS injuries [19].

\section{Pharmacokinetics and metabolism}

Multiple evidence suggests that the human body can utilize methylcobalamin more efficiently than cyanocobalamin and increase vitamin B12 coenzymes. The amount of methylcobalamin derived from a small oral dose is similar to cyanocobalamin, but there is increased cobalamin retention in the liver than with methylcobalamin. When in circulation in the plasma, this cobalamin is bound to two carrier proteins: transcobalamin (TC) and haptocorrin. Cobalamin transfer from the intestine into the blood and to the cells occurs with TC, a 43-kDa non-glycoprotein. Two components, total homocysteine (tHcy) and methylmalonic acid, act as functional markers for vitamin B12 status in adults [20]. Methylcobalamin excretion is through the urinary pathway and the amount excreted is one-third for the similar dose of cyanocobalamin, hence indicating more excellent cellular retention. Studies have shown that a dose of $0.5-6 \mathrm{mg} /$ day is sufficient for clinical effectiveness, and there is a therapeutic advantage in increasing the dose. A plethora of drug delivery systems have been evaluated for methylcobalamin administration and have been found to provide positive results [21]. 
Treatment for neuropathy and peripheral nerve injuries

Methylcobalamin has been used alone and in combination with other drugs to manage peripheral neuropathy, and multiple studies have been done on the same.

In a study by Li., et al. [22], 108 patients with diabetic neuropathies were randomized into two groups, the vitamin B12 group and methylcobalamin group, for 12 weeks. This was a single-blinded positive control study. At the end of the study period, patients exhibited significant improvement in the symptoms. In addition, they improved the nerve reflections and conduction velocity to a certain extent, with no considerable control group changes. In a study by Yoshioka., et al. [23], the effect of methylcobalamin on subclinical neuropathy of diabetic patients was also investigated utilizing power spectral analysis of heart rate variations. They found that after two months of methylcobalamin administration, 20 wellcontrolled diabetic patients showed a significant improvement of vibratory perception threshold and low-frequency components of power spectral density, implicating methylcobalamin's efficacy in peripheral diabetic and subclinical autonomic neuropathy.

Zhu., et al. [24] conducted a four-week study where methylcobalamin has been combined with prostaglandin E1 (PGE1) with 72 patients and demonstrated that along with a reduction in symptoms, there is significant amelioration of electrophysiological actions ( $\mathrm{p}<0.01$ ). De-Qi Jiang., et al. [25] conducted a randomized controlled study where Fasudil was combined with methylcobalamin for management of neuropathy and reported that the combination of the two yielded significantly improved results in patients ( $p<0.00001)$ than monotherapy with either. Manish., et al. [26] conducted a study to assess the effectiveness of fixed combinations of methylcobalamin, folic acid, biotin, benfotiamine, and pyridoxine. They concluded that the treatment led to a significant reduction from the baseline score for various neuropathic symptoms within four weeks. After 12 weeks, there was a $96 \%$ and $99.2 \%$ reduction in tingling and burning sensation, respectively, with no severe advents throughout the treatment period.

The peripheral nerve response to injury extends beyond the site of damage but involves neural cells in the spinal cord and ganglia. Schwann cells, macrophages, inflammatory cells, and neurotrophic factors are involved in this complex cascade [2]. The injured peripheral nerve attempts to compensate for the lost functions by strengthening and reprogramming the uninjured pathways [1]. The whole regeneration and repair process after peripheral nerve injury has not been completely understood. The peripheral nerves can regenerate after injury on their own due to the activation of their intrinsic growth capacity [2]. The sciatic nerve is commonly used for studying peripheral nerve regeneration since it consists of both sensory and motor neurons $[3,4]$. Vitamin B complex helps to alleviate degeneration in the nervous system, and vitamin B1 (thiamine), vitamin B6 (pyridoxine) in combination with vitamin B12 are clinically administered [27]. These vitamins, in particular vitamin B12, exhibit essential roles in various biological events to maintain normal neural functions [3,27]. Application of vitamin B complex or vitamin B12 has been shown to increase the number of myelinated nerve fibers, Schwann cells, and the diameter of axons, thereby promoting the regeneration of myelinated nerve fibers and the proliferation of Schwann cells [28]. Treatment with vitamin B complex or vitamin B12 increases the expression of brain-derived neurotrophic factor (BDNF) in the injured sciatic nerves at both mRNA and protein levels, but it does not affect the expression of glial cell-derived neurotrophic factor, neurotrophin-3, and interleukin-6; in addition, vitamin B complex and vitamin B12 have been shown to promote the regeneration and functional recovery of injured sciatic nerves through increasing BDNF expression [29]. Supplementation of vitamin B12 may enhance peripheral nerve regeneration [14]. High-dose vitamin B12 has been shown to have the potential to treat peripheral nerve injury [15].

\section{Safety and tolerability}

Throughout the plethora of studies conducted on methylcobalamin, most of them reported no adverse effects, including high dose intravenous methylcobalamin therapy. But a study by Manish., et al. [26], an overall global assessment, demonstrated that $97.8 \%$ of the cases showed excellent and $2.2 \%$ had good tolerability of the treatment of a combination of methylcobalamin (B12), folic acid (B9), biotin (B7), benfotiamine (B1) and pyridoxine (B6). The adverse effects included abdominal pain, nausea, and vomiting. But all these were self-limiting and resolved within the treatment period. It has been reported that, at very high doses, methylcobalamin can cause diarrhea, paresthesia, rhinitis, ataxia, pruritis, allergic reactions, and blood clots [30]. And it has been advised that patients with polycythemia should take care while taking this treatment as it can exaggerate the existing condition [31].

\section{Discussion}

The peripheral nerve response to injury extends beyond the site of damage but involves neural cells in the spinal cord and ganglia. Schwann cells, macrophages, inflammatory cells, and neurotrophic 
factors are involved in this complex cascade [2]. The injured peripheral nerve attempts to compensate for the lost functions by strengthening and reprogramming the uninjured pathways [1]. B vitamins such as thiamine (B1), pyridoxine (B6), and cyanocobalamin (B12) have antinociceptive effects in experimental animals with neuronal injury associated chronic and acute pain [32-34].

Vitamins B6 and B12 have been shown to counteract the deleterious effects of ischemia on neurons [35,36]. These vitamins play crucial roles in nutrition, nerve conduction, axonal transport, synthesis of neurotransmitters, and excitation via cyclic guanosine monophosphate (cGMP) signaling pathway [32]. Therefore, the reconstructive effect of vitamin $B$ complex on degenerative nerves may facilitate the recovery of nerve function after peripheral nerve injury. Resolution of peripheral nerve injury may benefit from a multi-nutrient treatment approach since B-complex vitamins are quintessential for optimum functioning of the nervous system [26]. The unavailability of these vitamins may decrease the enzymatic activity of some enzymes and result in neurotoxicity. Vitamin B12 acts as a scavenger of the reactive oxygen species and has a neuroprotective function owing to its anti-necrotic and anti-apoptotic effects on neurons [29,37]. Vitamin B12 increases the regeneration of axons, and the metabolic pathway of vitamin B12 is closely related to neuronal survival and repair after injury $[3,29,38]$. Improvement of B-complex vitamins delivery system may provide new eras for treating nerve injuries and yield new insights for understanding the pathophysiology. These vitamins may have synergistic effects that lead to the production of endogenous neurotropic factors, which enhance peripheral nerve repair [29]. Altun., et al. [39] conducted a study to evaluate whether tissue levels of vitamin B complex and vitamin B12 were altered after crush-induced peripheral nerve injury in an experimental rat model. The tissue level of vitamin B12 in the injured sciatic nerve was significantly lower at $1,6,12$, and 24 hours than in the control group. These results suggest that tissue levels of vitamin B complex and vitamin B12 vary with the progression of crush-induced peripheral nerve injury, and supplementation of these vitamins in the acute period may be beneficial for accelerating nerve regeneration.

From the above mentioned, it is evident that irrespective of being used in combination or as monotherapy, administering methylcobalamin in patients with traumatic nerve injury and neuropathy has significantly improved symptoms. Although the parenteral route can be utilized, there is not much data to support the fact that they can provide much improved results.
Along with the improvement in neurological symptoms, recent studies have suggested that they also have a role in reducing neuropathic pain. This comes as a glimmer of hope as other pain control methods in diabetic neuropathic pain are primarily ineffective and with severe side effects [40]. Studies are also being conducted on assessing the impact of methylcobalamin on pain in the lower back, trigeminal neuralgia, etc. [41-45].

\section{Conclusion}

From the above data, it can be concluded that methylcobalamin plays an essential role in the human body, whose deficiency, combined with other factors, will cause neurological symptoms and decreased peripheral nerve repair. Replenishment of the same leads to an alleviation of the symptoms and acceleration of repair. When given in mild doses for the long term, no adverse effects have yet been reported, and those which were reported rarely were managed in the treatment period itself. Along with providing good results in the long term, it sits significantly better than other conventional methods for the management of neuropathies and associated neuropathic pain.

\section{Bibliography}

1. Burnett MG and Zager EL. "Pathophysiology of peripheral nerve injury: a brief review". Neurosurgery Focus 16 (2004): 1-7.

2. Rishal I and Fainzilber M. "Retrograde signaling in axonal regeneration". Experimental Neurology 223 (2010): 5-10.

3. Hobbenaghi R., et al. "Neuropathological and neuroprotective features of vitamin B 12 on the dorsal spinal ganglion of rats after the experimental crush of sciatic nerve: an experimental study". Diagnostic Pathology 8 (2013): 1-8.

4. Bridge PM., et al. "Nerve crush injuries-a model for axonotmesis". Experimental Neurology 127 (1994): 284-290.

5. For Health Statistics NC. "National hospital ambulatory medical care survey: 2010 emergency department summary tables". Atlanta, GA Centers Dis Control Prev (2014).

6. Hughes R. "Investigation of peripheral neuropathy". BMJ 341 (2010).

7. Thakkar K and Billa G. "Treatment of vitamin B12 deficiency-Methylcobalamine? Cyancobalamine? Hydroxocobalamin?clearing the confusion". European Journal of Clinical Nutrition 69 (2015): 1-2. 
8. Zhang Y-F and Ning G. "Methylcobalamin". Expert Opinion on Investigational Drugs 17 (2008): 953-964.

9. Group Alsu. "ALSUntangled No. 30: Methylcobalamin". Amyotroph Lateral Scler Frontotemporal Degener 16 (2015): 536539.

10. Lexicomp. "Drug Information Handbook with International Trade Names Index". Lexi-Comp (2016).

11. Sun Y., et al. "Effectiveness of vitamin B12 on diabetic neuropathy: systematic review of clinical controlled trials". Acta Neurologica Taiwanica 14 (2005): 48-54.

12. Gupta JK and Sana QS. "Potential benefits of methylcobalamin: A review". Austin Journal of Pharmacology and Therapeutics 3 (2015): 1076.

13. Jayabalan B and Low LL. "Vitamin B supplementation for diabetic peripheral neuropathy". Singapore Medical Journal 57 (2016): 55.

14. Watanabe T., et al. "Ultra-high dose methylcobalamin promotes nerve regeneration in experimental acrylamide neuropathy". Journal of the Neurological Sciences 122 (1994): 140-143.

15. Okada K., et al. "Methylcobalamin increases Erk1/2 and Akt activities through the methylation cycle and promotes nerve regeneration in a rat sciatic nerve injury model". Experimental Neurology 222 (2010): 191-203.

16. Andrès E., et al. "Vitamin B12 (cobalamin) deficiency in elderly patients". CMAJ 171 (2004): 251-259.

17. Weir DG., et al. "The biochemical basis of the neuropathy in cobalamin deficiency". Baillière's Clinical Haematology 8 (1995): 479-497.

18. Liu KW., et al. "Metformin-related vitamin B12 deficiency". Age Ageing 35 (2006): 200-201.

19. Kikuchi M., et al. "Protective effects of methylcobalamin, a vitamin B12 analog, against glutamate-induced neurotoxicity in retinal cell culture". Investigative Ophthalmology and Visual Science 38 (1997): 848-854.

20. Obeid R., et al. "The cobalamin-binding proteins transcobalamin and haptocorrin in maternal and cord blood sera at birth". Clinical Chemistry 52 (2006): 263-269.
21. Yazaki Y., et al. "A single-center, double-blinded, randomized controlled study to evaluate the relative efficacy of sublingual and oral vitamin B-complex administration in reducing total serum homocysteine levels". Journal of Alternative and Complementary Medicine 12 (2006): 881-885.

22. Li G. "Effect of methylcobalamin on diabetic neuropathies. Beijing methycobal clinical trial collaborative group". Zhonghua nei ke za zhi 38 (1999): 14-17.

23. Yoshioka $\mathrm{K}$ and Tanaka K. "Effect of methylcobalamin on diabetic autonomic neuropathy as assessed by power spectral analysis of heart rate variations". Hormone and Metabolic Research: Horm Stoffwechselforschung= Horm Metab 27 (1995): 43-44.

24. Zhu XP and Zhou ZG. "Clinical observation of combined therapeutic effect of prostaglandin E1 and methylcobalamin on diabetic peripheral neuropathy". Hunan yi ke da xue xue bao= Hunan yike daxue xuebao= Bull Hunan Med Univ 26 (2001): 343-344.

25. Jiang D-Q., et al. "Fasudil combined with methylcobalamin or lipoic acid can improve the nerve conduction velocity in patients with diabetic peripheral neuropathy: a meta-analysis". Medicine (Baltimore) 97 (2018).

26. Maladkar M., et al. "Post-marketing surveillance of fixed dose combination of methylcobalamin, alpha lipoic acid, folic acid, biotin, benfotiamine and vitamin B6-nutripathy for the management of peripheral neuropathy". Journal of Diabetes Mellitus 4 (2014): 124.

27. Jolivalt CG., et al. "B vitamins alleviate indices of neuropathic pain in diabetic rats". European Journal of Pharmacology 612 (2009): 41-47.

28. Lopatina T., et al. "Adipose-derived stem cells stimulate regeneration of peripheral nerves: BDNF secreted by these cells promotes nerve healing and axon growth de novo". PLoS One 6 (2011): e17899

29. Sun H., et al. "Dexamethasone and vitamin B12 synergistically promote peripheral nerve regeneration in rats by upregulating the expression of brain-derived neurotrophic factor". Archives of Medical Science 8 (2012): 924.

30. Maron BA and Loscalzo J. "The treatment of hyperhomocysteinemia". Annual Review of Medicine 60 (2009): 39-54. 
31. Partanen J., et al. "Natural history of peripheral neuropathy in patients with non-insulin-dependent diabetes mellitus". The New England Journal of Medicine 333 (1995): 89-94.

32. Wang Z-B., et al. "Thiamine, pyridoxine, cyanocobalamin and their combination inhibit thermal, but not mechanical hyperalgesia in rats with primary sensory neuron injury". Pain 114 (2005): 266-277.

33. Caram-Salas NL., et al. "Thiamine and cyanocobalamin relieve neuropathic pain in rats: synergy with dexamethasone". Pharmacology 77 (2006): 53-62.

34. Yu C-Z., et al. "Systematic administration of B vitamins attenuates neuropathic hyperalgesia and reduces spinal neuron injury following temporary spinal cord ischaemia in rats". European Journal of Pain 18 (2014): 76-85.

35. Hung K-L., et al. “Cyanocobalamin, vitamin B12, depresses glutamate release through inhibition of voltage-dependent $\mathrm{Ca} 2+$ influx in rat cerebrocortical nerve terminals (synaptosomes)". European Journal of Pharmacology 602 (2009): 230-237.

36. Kaneda K., et al. "Effects of B vitamins on glutamate-induced neurotoxicity in retinal cultures". European Journal of Pharmacology 322 (1997): 259-264.

37. Liao W-C., et al. "Methylcobalamin facilitates collateral sprouting of donor axons and innervation of recipient muscle in endto-side neurorrhaphy in rats". PLoS One 8 (2013): e76302.

38. Dominguez JC., et al. "A prospective, open label, 24-week trial of methylcobalamin in the treatment of diabetic polyneuropathy". Journal of Diabetes Mellitus 2.4 (2012).

39. Altun I and Kurutaş EB. "Vitamin B complex and vitamin B12 levels after peripheral nerve injury". Neural Regeneration Research 11 (2016): 842.

40. Zhang M., et al. "Methylcobalamin: a potential vitamin of pain killer". Neural Plasticity (2013).

41. Teramoto J. "Effects of Methylcobalamin on neuralgia”. Neurology Therapy 1 (1984): 315.

42. Singh PM., et al. "Analgesic efficacy and safety of medical therapy alone vs combined medical therapy and extraoral glossopharyngeal nerve block in glossopharyngeal neuralgia". Pain Medicine 14 (2013): 93-102.

43. Xu G., et al. "A single-center randomized controlled trial of local methylcobalamin injection for subacute herpetic neuralgia”. Pain Medicine 14 (2013): 884-894.
44. Hanai IY., et al. "Clinical study of methylcobalamin on cervicales”. Drug Therapy (NY) 13 (1980): 29.

45. Waikakul W and Waikakul S. "Methylcobalamin as an adjuvant medication in conservative treatment of lumbar spinal stenosis". Journal of the Medical Association of Thailand 83 (2000): 825-831.

\section{Volume 5 Issue 8 August 2021}

(C) All rights are reserved by Annamalai Thangavelu., et al. 